\title{
Nonlinear Internal Model Control Using Neural Networks and Fuzzy Logic: Application to an Electromechanical Process
}

\author{
Rodolfo E. Haber, ${ }^{1,2}$ J.R. Alique ${ }^{1}$, A. Alique ${ }^{1}$, and R.H. Haber ${ }^{3}$ \\ ${ }^{1}$ Instituto de Automática Industrial (CSIC). \\ km. 22,800 N-III, La Poveda. 28500 Madrid. \\ SPAIN. \\ rhaber@iai.csic.es \\ ${ }^{2}$ Escuela Técnica Superior \\ Universidad Autónoma de Madrid. \\ Ciudad Universitaria de Cantoblanco. \\ Ctra. de Colmenar Viejo, km 15. 28049 Madrid \\ SPAIN. \\ ${ }^{3}$ Departamento de Control Automático. \\ Universidad de Oriente. 90400. \\ CUBA
}

\begin{abstract}
This study explores the use of the internal-model control (IMC) paradigm using artificial neural networks (ANNs) and fuzzy logic (FL) to consider a force-control problem involving a complex electromechanical system, represented here by the machining process. The main goal is to control a single output variable, cutting force, by changing a single input variable, feed rate. This scheme consists of a dynamic model using ANNs to estimate process output and a fuzzy-logic controller (FLC) with the same static gain as the inverse model to determine the control inputs (feed rate) necessary to keep the cutting force constant. Three approaches, the fuzzy-logic controller (FLC), the internal-model controller (IMC) and a neuro-fuzzy controller (NFC), are simulated and their performances are assessed in terms of several performance measurements. The results demonstrate that the NFC strategy provides better disturbance rejection than IMC and FLC for the cases analysed.
\end{abstract}

\section{Introduction}

Nowadays artificial neural networks (ANNs) and fuzzy logic (FL) are probably the most widely used artificial intelligence techniques in model identification and controlsystem design. ANNs have shown an excellent capability to model any non-linear function to a desired degree of accuracy [1]. Because of this property, ANNs are suitable for the identification and control of non-linear plants [2,3]. Of all the different classes of networks, feedforward neural networks and particularly multi-layer

* Corresponding author

P.M.A. Sloot et al. (Eds.): ICCS 2003, LNCS 2657, pp. 351-360, 2003.

(c) Springer-Verlag Berlin Heidelberg 2003 
perceptrons (MLPs) are the most frequently used for non-linear control. Furthermore, FL has proven useful in control and industrial engineering as a very practical optimising tool. The growing number of successful industrial applications of fuzzy logic worldwide really provides the best credentials the technique could hope for [4]. Through FL we can plug human knowledge, expressed in qualitative terms, into control systems to provide us with an alternative mathematical tool for computation, human reasoning, and the integration of qualitative and quantitative information.

Combining different AI approaches in the control field has yielded interesting and efficient applications [5]. This paper pursues the same line and presents some preliminary results regarding a control strategy that is a useful synergy of a dynamic neural network trained from real-life data and used to predict process output and a fuzzy-logic control (FLC) that enhances the overall performance of the closed-loop system.

Internal-model control (IMC) is a well-established approach to controller design in which the process model is explicitly used in the control-system design procedure [6,7]. However, when an exact mathematical model is not available or is very sophisticated, IMC can get around the lack of an explicit plant model by using an experimental identification scheme. In such cases, ANNs can be used to produce an efficient control scheme based on the IMC principle. The IMC-based ANNs consists in training a network to learn the process' dynamics. Another ANN can be trained to learn the inverse dynamics so that it can be used as a non-linear controller [8]. If we can synthesise an FLC with a static gain equal to the static gain of the inverse model, and if the control system is stable with this controller, we obtain the offset-free control for constant setpoints and output disturbances [9]. The neuro-fuzzy control (NFC) scheme proposed here is inspired by IMC-based ANNs. The inverse of the dynamic ANNs is replaced in the forward path by a fuzzy controller, aiming at improving disturbance-rejection capability.

In this paper a complex electromechanical process, the machining process, is used as the test bed [10]. The electrical portion of the system includes DC and AC rotational motors, amplifiers, sensors and other components. The mechanical portion includes the rigid structure and the body with its different shafts, gears and reducer. The main goal is to implement machining-process optimisation through controlling a single output variable, the cutting force, by changing a single input variable, the feed rate. The effectiveness of the NFC scheme is demonstrated through simulations. The comparison of FLC, IMC and NFC is assessed using several performance criteria based on the given simulation results. The NFC can be inferred to make the control system more effective in disturbance rejection.

This paper is organized as follows. Section 2 introduces the machining-process models used in simulations. Section 3 explains the fuzzy approach in a simple feedback system to control the cutting force. Section 4 presents the design of an IMC based on ANNs. Section 5 addresses the NFC system's design and the comparison of the three control systems based on simulation results. The final section draws a number of conclusions. 


\section{The Machining Process}

The characteristics of the machining process as a complex electromechanical process severely limit the use of classic mathematical tools for modelling and control. Even so, a classic mathematical model could provide a characterisation of the dynamic behaviour of the machining process that could help to investigate and analyse machine-tool performance and limitations. The dynamics of the milling process (cutting-force response to changes in feed rate) can be approximately modelled using at least a second-order differential equation.

The following linear model is suggested in [11]:

$$
G_{L U}(z)=\frac{0.019 z+0.017}{z^{2}-1.75 z+0.77} .
$$

Another model obtained using second-order differential equations [12] is

$$
G_{R S}(z)=\frac{0.052 z+0.04}{z^{2}-1.42 z+0.45}
$$

The structure of a first-order cutting-force process including cutting speeds and non-linear depth-of-cut effects is proposed [13] thus:

$$
G_{L A}(z)=\frac{0.11}{z-0.85} a^{0.65} f^{0.63}
$$

$G_{P}=\left\{G_{L U}(z), G_{R S}(z), G_{L A}(z)\right\}$ represents the machining process from the classical viewpoint. Equations (1)-(3) are only valid over a narrow range; hence they cannot trespass certain limits in representing the process' complexity and uncertainty. However, they do provide a rough characterisation of the dynamic behaviour of the machining process and are used in Section 5 for simulations.

\section{Fuzzy Approach to Controlling Cutting Force}

The design of a fuzzy-logic controller (FLC) as detailed in [4] is introduced in this section. The input variables are the cutting-force error $\Delta F$ and the change in cuttingforce error $\Delta^{2} F$. The manipulated (action) variable selected is the feed-rate increment $\Delta f$, whereas the spindle speed is considered constant and preset by the operator. The control scheme is depicted in Fig. 1.

The controller output is inferred by means of the compositional rule. The SupProduct compositional operator was selected for the compositional rule of inference. For instance, applying the $T_{2}$ norm (product) and applying $S_{1}$ s-norm (maximum) yields (4). 


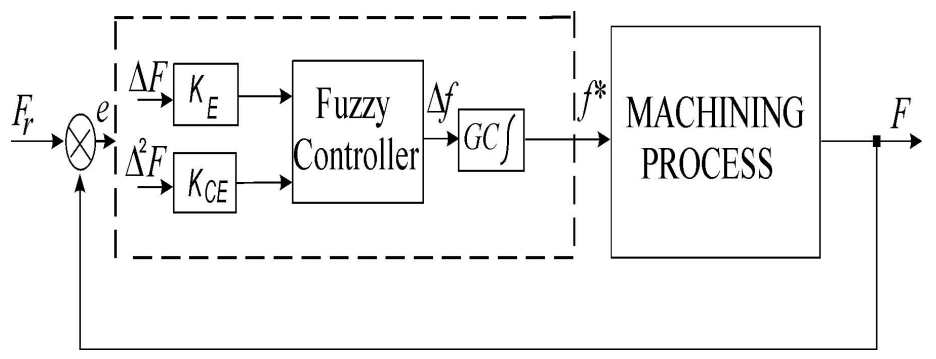

Fig. 1. Fuzzy-control scheme for the machining process.

$$
\mu\left(\Delta F, \Delta^{2} F, \Delta f\right)={\stackrel{m}{S_{1}}}_{i=1}^{m}\left[T_{2}\left[\mu_{\Delta F_{i}}(\Delta F), \mu_{\Delta^{2} F_{i}}\left(\Delta^{2} F\right), \mu_{\Delta f_{i}}(\Delta f)\right]\right]
$$

where $T_{2}$ represents the algebraic-product operation and $S_{1}$ represents the union operation $(\max ), m \times n=49$ rules. The crisp controller output, used to change the machine-table feed rate, is obtained by defuzzification employing the centre of area (COA) method defined as

$$
\Delta f=\frac{\sum_{\mathrm{i}} \mu_{R}\left(\Delta f_{i}\right) \cdot \Delta f_{i}}{\sum_{i} \mu_{R}\left(\Delta f_{i}\right)} .
$$

where $\Delta f$ is the crisp value of $\Delta f_{i}$ for a given crisp input $\left(\Delta F_{i}, \Delta^{2} F_{i}\right)$.

The strategy used to compute $f$ determines what type of fuzzy regulator is to be used. In this case it is a PI regulator:

$$
f *(k)=f(k-1)+\Delta f(k) .
$$

\section{IMC System Based on Neural Networks}

First an ANN is trained to learn the dynamics of the process and is therefore given known input and output data sets. Training with real-time data uses data obtained from actual machining operations. So, one of the neural-network models developed in [10] is selected as a basis for IMC control. The dynamic equation can be described in reduced notation by

$$
\hat{F}(t)=G_{M}(\mathbf{F}, \mathbf{f}) .
$$

where $G_{M}$ is an unknown function to be identified, $F$ is the cutting force exerted during the removal of metal chips and $f$ is the relative feed speed between tool and worktable. Cast in vector form, $\mathbf{f}$ and $\mathbf{F}$ are the input and output respectively defined 
as $\mathbf{F}=\left[\begin{array}{lll}F(t-1) & \cdots & F(t-n)\end{array}\right]$ and $\mathbf{f}=\left[\begin{array}{ll}f(t-1), & \cdots, f(t-m)\end{array}\right], t$ is the discrete time instant and $n, m \in Z$ A successful identification scheme should insure $\hat{F}(t)$ values as close as possible to those of $F(t)$ (actual output).

The training algorithm was developed using MATLAB. The topology was initially chosen as follows: one input $f$ and one output $\hat{F}$, a linear activation function at the output and one hidden layer using the hyperbolic tangent for the activation function. The type of model was selected using a priori knowledge of the milling process and the types of models considered in previous work. An ANN with four neurons in the input, four neurons in the hidden layer and one in the output layer was selected. The dynamic equation can be described in reduced notation by

$$
\hat{F}(t+1)=G_{M}(f(t) \quad f(t-1) F(t) F(t-1)) .
$$

where $G_{M}=\left\{\mathbf{w}_{\mathbf{f}}, \mathbf{W}_{\mathbf{f}}\right\}$,

$$
\begin{gathered}
\mathrm{W}_{\mathrm{f}}=\left[\begin{array}{ccccc}
-0.4355 & 0.0901 & 0.7938 & -0.5570 & -0.4723 \\
0.3790 & 0.3895 & -0.2630 & -0.1079 & 0.1944 \\
0.5686 & -0.3144 & -0.7093 & 0.8608 & 0.1641 \\
-0.2621 & 0.7771 & -0.3636 & 0.4915 & 0.9354
\end{array}\right] \\
\mathrm{W}_{\mathrm{f}}=\left[\begin{array}{lllll}
-0.6189 & 0.2987 & 1.0114 & 1.1728 & -1.2777
\end{array}\right]
\end{gathered}
$$

The inverse model was obtained on the basis of generalised training [8]. Therefore, the network is trained off-line to minimise

$$
J(\theta)=\sum_{t=1}^{M}(f(t)-\hat{f}(t))^{2}
$$

Another ANN is trained to learn the inverse dynamics of the process and to work as a non-linear controller. The dynamic equation of the inverse model can be described in reduced notation by

$$
f(t)=G_{M}^{\prime}(F(t+1) F(t) f(t-1) \quad f(t-2)) .
$$

where $G_{M}^{\prime}=\left\{\mathbf{w}_{\mathbf{i}}, \mathbf{W}_{\mathbf{i}}\right\}$,

$$
\mathrm{w}_{\mathrm{i}}=\left[\begin{array}{ccccc}
0.1020 & -0.0190 & -0.0096 & -0.1468 & 0.0670 \\
1.5430 & 0.6541 & -0.2669 & -0.7483 & 0.2376 \\
0.1216 & -0.1247 & 0.0590 & 0.2472 & -1.1422 \\
0.1328 & -0.0265 & -0.0356 & 0.1701 & 0.5677
\end{array}\right] \text {. }
$$




$$
\mathrm{W}_{\mathrm{i}}=\left[\begin{array}{lllll}
-3.3957 & 0.0569 & 2.0374 & 2.7758 & 0.4598
\end{array}\right] .
$$

The bias vector (term) is represented by the last column of (9)-(10) and (13)-(14).

a)

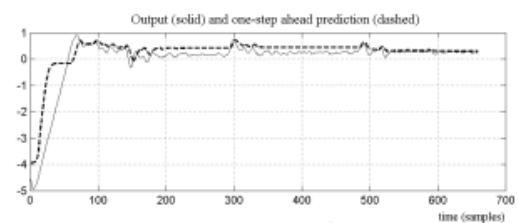

Prediction error $(\hat{F}, \hat{F})$

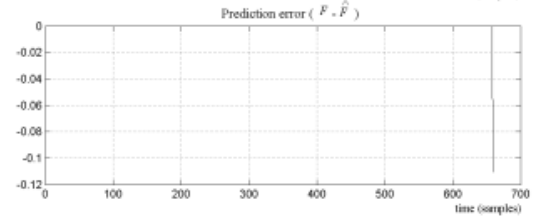

Fig. 2. a) Comparison between plant and model outputs, (b) Comparison between actual control signal and prediction.

The result of the forward model for predicting cutting force in real time is shown in Figure 2a. The behaviour of the inverse model is depicted in Figure 2b. Several performance indices were used to evaluate the forward model and the inverse model, defined as

$$
\begin{gathered}
N S S E=\sqrt{\frac{S S E}{2 N}} . \\
F P E=\frac{N S S E(N+p)}{N-p} . \\
E N V=\frac{2 \cdot N \cdot N S S E}{N-p} . \\
S B C=N \cdot \log (S S E / N)+p \cdot \log (N) .
\end{gathered}
$$

where $S S E$ is the sum of squared errors, NSSE is the prediction error, FPE is the final prediction error, $p$ is the effective number of weights and $N$ is the total number of samples.

$N S S E_{\mathrm{f}}=0.37, F P E_{\mathrm{f}}=0.39, E N V_{\mathrm{f}}=0.77$ and $S B C_{\mathrm{f}}=-3942.7$ were yielded for the forward model. $N S S E_{\mathrm{i}}=0.0274, F P E_{\mathrm{i}}=0.0291, E N V_{\mathrm{i}}=0.0564$ and $S B C_{\mathrm{i}}=-2831.2$ were yielded for the inverse model. 


\section{Simulations and Results}

Models (1)-(3) were used in a feedback-control structure as depicted in Figure 3. The fuzzy-control surface with the same static gain as the inverse neural model $G_{M}$ ' is shown in Figure 4.

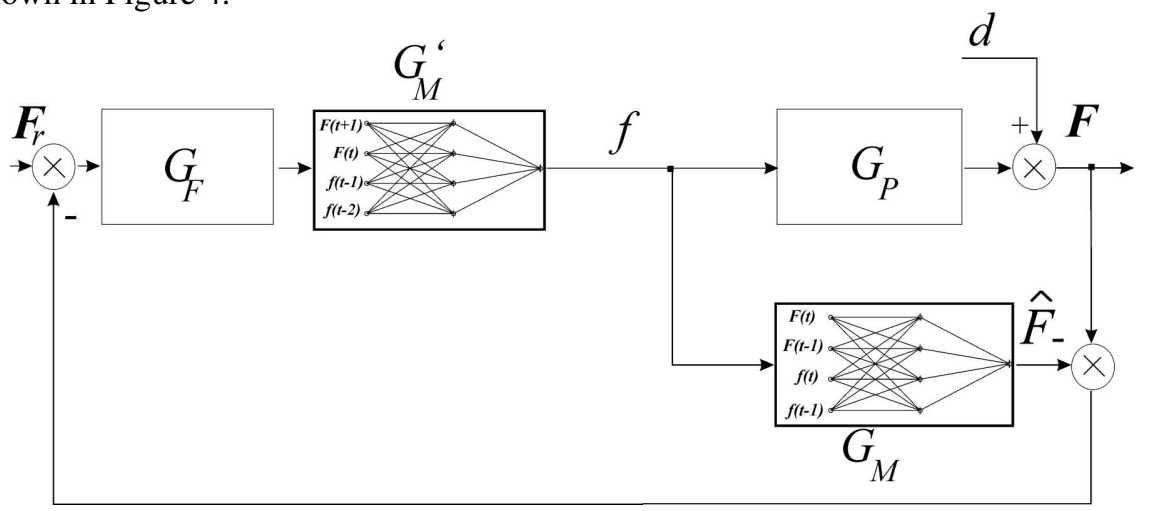

a)

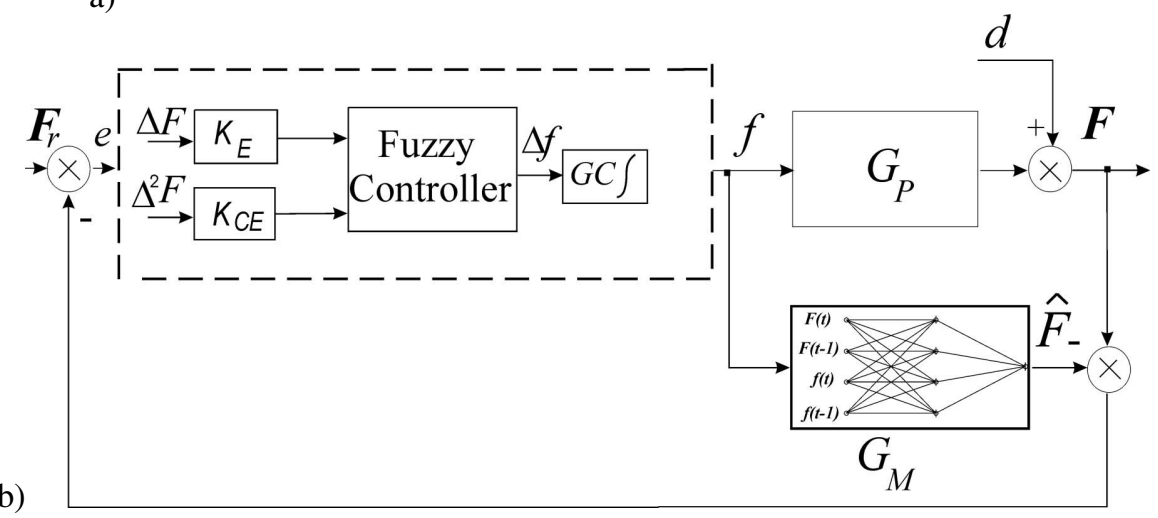

Fig. 3. a) IMC scheme, b) NFC diagram.

In the sequel, simulations were run based on linearised plant models (1)-(3) representing approximate process models and using the control schemes depicted in Figures 1 and 3 .

The inclusion of a filter, $G_{F}$ (see Fig. 3 a), was found to reduce the high-frequency gain and hence improve the robustness of the system. The filter also smoothes out noisy/rapidly changing signals, reducing the transient response of the controller. Therefore robustness is considered explicitly in the IMC design, although it can be adjusted by choosing filter $G_{F}$ accordingly.

A common choice from classical literature is the low-pass filter. Using the $z-$ transform $G_{F}(z)$ is given by 


$$
G_{F}(z)=\frac{1-k_{2}}{z-k_{1}} .
$$

where $k_{1}, k_{2}$ are design parameters and usually $k_{1}=k_{2}$.

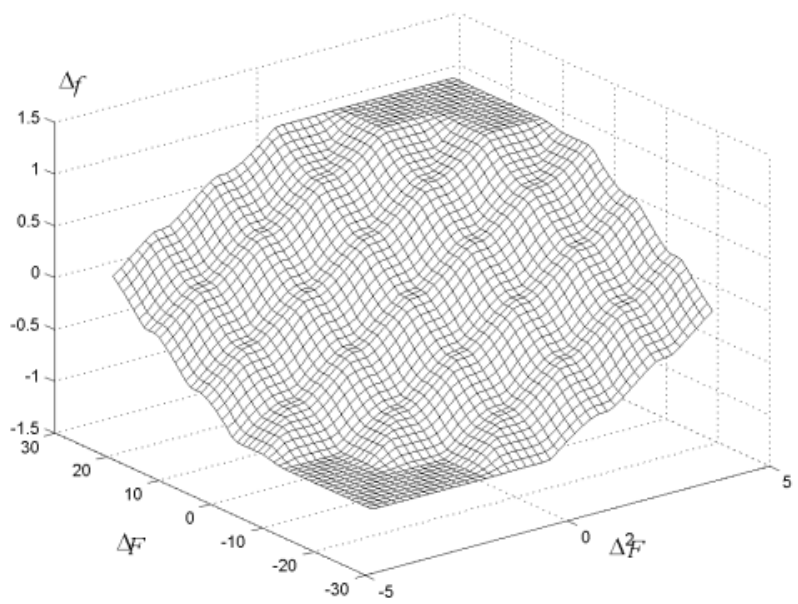

Fig. 4. Fuzzy-control surface for the NFC scheme.

In order to analyse the disturbance-rejection capabilities of the control system, additive noise plus the influence of unmodelled dynamics are considered in order to estimate the dynamics that can be expected in real-time applications. The following additive noise is assumed to corrupt the output:

$$
d(t)=0.1 \cdot(\sin 8 t+\sin 12 t+\sin 23.66 t+\sin 35.49 t) .
$$

Now, the more realistic model of the process, including unmodelled multiplicative dynamics plus (20), is represented by

$$
G_{P}(z)=G_{*}(z) \cdot \frac{0.095}{z-0.904}+d(z) .
$$

where $G_{*}(z)$ is an ideal process model represented by (1)-(3).

Figure 5 shows the simulation results with and without the influence of unmodelled dynamics and disturbances. In order to evaluate the simulation results, various performance indices were calculated such as integral absolute error (IAE), integral square error (ISE) and integral of time-weighted absolute error (ITAE). The overshoot, $M_{p t}$, was also computed.

A comparison of FLC, IMC and NFC is shown in Table 1. Both FLC and IMC are able to regulate the process in the presence of noise. FLC gives a slightly better transient response but more error and a bigger overshoot. The resulting NFC performs well in both transient and steady-state conditions. 


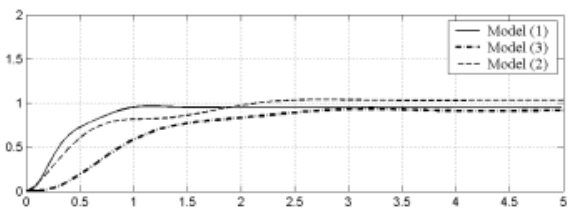

a)

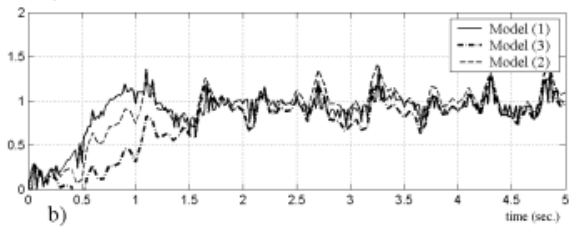

a)

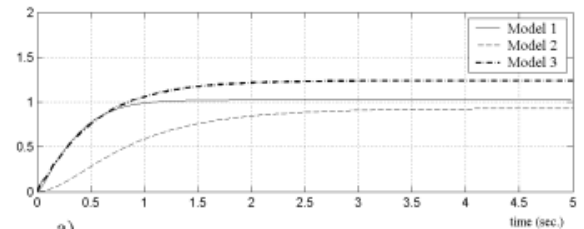

a)

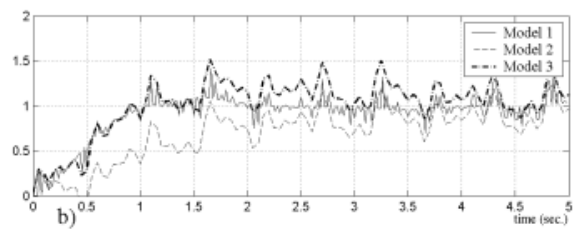

b)

Fig. 5. Closed-loop response using (a) IMC, (b) NFC.

Table 1. Control strategy comparison.

\begin{tabular}{cccccccccccccccc}
\hline \multicolumn{1}{c}{ Criterion } & \multicolumn{3}{c}{ ISE } & \multicolumn{3}{c}{ IAE } & \multicolumn{3}{c}{ ITAE } & & \multicolumn{2}{c}{$M_{p t}[\%]$} \\
\hline Model & FLC & IMC & NFC & FLC & IMC & NFC & FLC & IMC & NFC & FLC & IMC & NFC \\
\hline (1) & 12.4 & 20.0 & 10.5 & 21.9 & 28.5 & 21.5 & 12.7 & 31.7 & 18.1 & NO & NO & 2.41 \\
\hline$(2)$ & 13.9 & 15.4 & 13.8 & 20.2 & 30.4 & 29.0 & 16.9 & 57.4 & 53.4 & NO & NO & NO \\
\hline$(3)$ & 7.1 & 7.1 & 11.1 & 13.0 & 16.1 & 36.3 & 10.8 & 23.8 & 130.3 & 1.2 & 4.0 & 23.9 \\
\hline$(21)-(1)$ & 43.3 & 20.7 & 18.4 & 90.2 & 43.4 & 38.0 & 180.8 & 64.7 & 50.9 & 85.2 & 35.9 & 41.2 \\
\hline$(21)-(2)$ & 25.6 & 20.4 & 19.8 & 48.7 & 38.2 & 39.4 & 136.6 & 88.0 & 90.1 & 61.4 & 27.7 & 27.3 \\
\hline$(21)-(3)$ & 20.6 & 11.9 & 11.1 & 44.6 & 27.5 & 27.9 & 140.1 & 66.6 & 73.0 & 65.3 & 41.3 & 51.6 \\
\hline
\end{tabular}

\section{Conclusions}

This paper presents some preliminary results of a control strategy based on the internal-model control paradigm, which is a useful synergy of a dynamic ANN trained from real-life data and used to predict process output and a fuzzy-logic control (FLC) that enhances the control system's overall performance. The proposed neuro-fuzzy control (NFC) scheme is inspired by the non-linear IMC; the inverse model represented by a dynamic ANN is replaced by a fuzzy controller in order to improve disturbance-rejection capability. A dynamic ANN is trained to learn the process 
dynamics and another ANN is trained to learn the inverse dynamics. An FLC with the same static gain as the inverse model is used in the forward path of the control loop.

The results shown here are still preliminary because only linear-process models have been considered for simulation. Nevertheless, the simulation tests show that NFC performs better than FLC and IMC on the basis of ISE, IAE and ITAE performance measurements. However, FLC's simplicity and satisfactory transient response can make this scheme a good one for dealing with strict time-computing requirements.

In this paper the ANN was trained off-line using actual data, but in future on-line training as well as the real-time application of the NFC will be implemented in order to enable adaptation and check actual plant behaviour.

\section{References}

1. Hornik K., Stincheombe M., White H.: Multilayer feedforward networks are universal approximators. Neural Networks 2 (1989) 359-366

2. Hunt K.J., Sbarbaro D., Zbikowski R., Gawthrop P.J.: Neural Networks for control systems- A survey. Automatica 28(6) (1992) 1083-1112

3. Nelles O.: Nonlinear system identification: From classical approaches to neural networks and fuzzy logic, Springer Verlag (2000)

4. Haber R.E., Haber R.H., Alique A., Ros S.: Application of knowledge-based systems for supervision and control of machining processes. In: S.K. Chang (ed.): Handbook of Software Engineering and Knowledge Engineering Vol. II. World Scientific Publishing (2002) 327-362

5. Hung S.J., Lian R.J.: A hybrid fuzzy logic and neural networks algorithm for robot motion control. IEEE Transactions on Industrial Electronics 44(3) (2000) 217-229

6. Morari M., Zafiriou E.: Robust process control. Prentice Hall, Englewood Cliffs, NJ, (1989)

7. Goodwin G.C., Graebe S.F., Salgado M.E.: Control System Design. Prentice Hall, NJ, USA, pp. 567-582 (2001)

8. Norgard M., Ravn O., Poulsen N.K., Hansen L.K.: Neural networks for modelling and control of dynamics systems, Springer-Verlag, London, (2000)

9. Rivals I., Personnaz L.: Nonlinear internal model control using neural networks: Application to processes with delay and design issues. IEEE Transactions on Neural Networks 11(1) (2000) 80-90

10. Haber R.E., Haber R.H., Ros S., Alique A., Alique J.R.: Dynamic model of the machining process on the basis of neural networks: From simulation to real time application. Lecture Notes in Computer Science 2331 (2002) 574-583

11. Lauderbaugh L.K., Ulsoy A.G.: Model reference adaptive force control in milling. ASME Journal Engineering of Industry 111 (1989) 13-21

12. Rober S.J., Shin Y.C.: Control of cutting force for milling processes using an extended model reference adaptive control scheme. Journal of Manufacturing Science and Engineering 118 (1996) 339-347

13. Landers R., Ulsoy A.: Model-based machining control, ASME Journal of Dynamics Systems, Measurement and Control 122(3) (2000) 521-527 